\title{
Velocity dependence of atomic-scale friction: a comparative study of the one- and two-dimensional Tomlinson model
}

\author{
C. Fusco ${ }^{1 *}$ and A. Fasolino ${ }^{1,2}$ \\ 1 Solid State Theory, IMM, Radboud University Nijmegen, \\ Toernooiveld 1, 6525 ED Nijmegen, The Netherlands \\ ${ }^{2}$ HIMS/WZI, Faculty of Science, University of Amsterdam, \\ Nieuwe Achtergracht 1661018 WV Amsterdam, The Netherlands
}

(August 24, 2018)

\begin{abstract}
We present a comparative analysis of the velocity dependence of atomic-scale friction for the Tomlinson model, at zero and finite temperatures, in $1 D$ and $2 D$, and for different values of the damping. Combining analytical arguments with numerical simulations, we show that an appreciable velocity dependence of the kinetic friction force $F_{\text {fric }}$, for small scanning velocities $v_{s}$ (from $1 \mathrm{~nm} / \mathrm{s}$ to $2 \mu \mathrm{m} / \mathrm{s}$ ), is inherent in the Tomlinson model. In the absence of thermal fluctuations in the stick-slip regime, it has the form of a power-law, $F_{\text {fric }}-F_{0} \propto v_{s}^{\beta}$ with $\beta=2 / 3$, irrespective of dimensionality and value of the damping. Since thermal fluctuations enhance the velocity dependence of friction, we provide guidelines to establish when thermal effects are important and to which extent the surface corrugation affects the velocity dependence.

68.35.Af, 68.37.Ps, 46.55.+d
\end{abstract}

\section{INTRODUCTION}

Although friction is a common phenomenon in everyday experience, the fundamental mechanisms governing friction at the atomic level are still under discussion. For macroscopic contacts the friction force is found to be independent of the sliding velocity, but no consensus has been reached on the velocity dependence at the nanometer scale. A very powerful technique for measuring atomic-scale friction is provided by Atomic Force Microscopy (AFM) [1,2]. Since scanning velocities accessible by AFM are very small, typically from $\mathrm{nm} / \mathrm{s}$ to few $\mu \mathrm{m} / \mathrm{s}$, it is relevant to study friction dynamics in this regime. Velocity dependence of friction is relevant both for applications and from a fundamental point of view, and has been discussed in several AFM [3-11] and Quartz Crystal Microbalance [12] experimental studies as well as theoretical works [9-11,13-19]. Depending on the investigated systems and on the experimental conditions, different and somewhat contradictory results for the velocity dependence have been found. In the original experiments of Mate et al. [3] the authors state that the frictional forces of a tungsten tip on graphite show little dependence on velocity for scanning velocities up to $400 \mathrm{~nm} / \mathrm{s}$. A similar behavior up to velocities of several $\mu \mathrm{m} / \mathrm{s}$ has been reported also in the work of Zwörner et al. [10], where friction on different carbon structures has been studied. The authors of Ref. [10] claim that a $1 D$ Tomlinson model at $T=0$ can reproduce a velocity independent friction force for scanning velocities up to $\sim 1$ $\mu \mathrm{m} / \mathrm{s}$, while giving a linear increase of friction for higher velocities. At variance with the $1 D$ case, in the $2 D$ version of the Tomlinson model at $T=0$, which has been recently analyzed by Prioli et al. [11], a smooth increase of friction for velocities lower than $\sim 300 \mathrm{~nm} / \mathrm{s}$ has been found. In view of the results of Zwörner et al. for the $1 D$ case, the authors argue that this effect should be peculiar of the $2 D$ model, due to the non-linear coupling between the two degrees of freedom in the system. The role of damping has not been addressed in Refs. [10,11]. In the underdamped regime, the velocity dependence can be quite complex, especially at intermediate-large velocities, where the system displays bifurcations, chaotic motion, resonances and hysteresis [14]. In the overdamped regime, Robbins and Müser [20] suggest velocity independent friction.

An increase of the friction force has been observed for small velocities also in Refs. $[6,7,9]$ and it has been attributed to thermally activated processes $[6,7,9,19]$. By means of a simple thermal activation probabilistic analysis in $1 D$, Gnecco et al. [9] have obtained a logarithmic increase of friction with scanning velocity which fits their experimental data quite well. A similar dependence had been obtained using a simple stress-modified thermallyactivated Eyring model [6]. In a recent work, Sang et al. [19] have corrected this logarithmic relation at not too small velocities: they propose a $\left|\ln v_{s}\right|^{2 / 3}$ dependence of the friction force, where $v_{s}$ is the scanning velocity. However, recent experiments showing an increase of friction with velocity [11] do not display the logarithmic behavior related to thermal activation, but rather suggest an athermal power-law $v_{s}^{\beta}$ behavior, as found in related sys-

*Author to whom correspondence should be addressed. Electronic address: c.fusco@science.ru.nl. 
tems, such as charge density waves [21] and in boundary lubrication [22].

In view of the contradictory results presented above, here we reexamine this issue for Tomlinson-like models in $1 D$ and $2 D$, for different values of the damping, and both with and without thermal effects. In particular, we focus on the importance of the athermal contribution to the velocity dependence of friction, which is intrinsically present in the Tomlinson model. We show by means of a combined analytical and numerical analysis that the exponent $\beta$ is independent of the spatial dimension and of the damping. Then we discuss the role of thermal fluctuations, establishing guiding rules to understand where thermal effects become dominant.

In Sec. II we illustrate the model studied and the numerical techniques. In Sec. III we discuss the results for the athermal velocity dependence of friction and in Sec. IV we include thermal fluctuations. Finally, we present some concluding remarks in Sec. V.

\section{MODEL}

The Tomlinson model [23] has been successfully used to describe the motion of a tip and to model the scan process in AFM [24-27]. In particular, this model can reproduce the stick-slip motion observed in experiments and can be used to study frictional dynamics. Here we consider the $1 D$ Tomlinson model and its extension in $2 D$ at $T=0$ and $T \neq 0$. A cantilever tip of mass $m$ interacts with the surface via a periodic potential $V_{t s}$ and is attached by a spring of elastic constant $k_{x}$ to a support moving at constant velocity $v_{s}$ along the $x$ direction. For the $1 D$ case we choose $V_{t s}$ of the form

$$
V_{t s}(x)=V_{0}\left[1-\cos \left(2 \pi x / a_{x}\right)\right] \text {, }
$$

where $a_{x}$ is the lattice constant of the substrate. The elastic interaction between the tip and the support is

$$
V_{e l}(x)=\frac{1}{2} k_{x}\left(x-x_{s}\right)^{2},
$$

where the support position $x_{s}$ is

$$
x_{s}=v_{s} t
$$

It is assumed that the tip is a point-like object, representing the average over many atoms of the real tip-surface contact. Energy dissipation in this model is introduced by adding a damping term proportional to the tip velocity in the equation of motion. Thermal fluctuations are taken into account by a stochastic force, in the framework of the Langevin approach. Thus, the equation of motion in $1 D$ becomes

$$
m \ddot{x}+m \eta \dot{x}+\frac{2 \pi V_{0}}{a_{x}} \sin \left(\frac{2 \pi x}{a_{x}}\right)+k_{x}\left(x-v_{s} t\right)=f(t),
$$

with the random force $f(t)$ satisfying the conditions $<f(t)>=0$ and $<f(t) f(0)>=2 m \eta k_{B} T \delta(t)$, where $<\cdot>$ indicates an ensemble average, $\eta$ is the damping parameter and $k_{B}$ is the Boltzmann's constant [28]. The static friction force in this model is simply given by the force needed to overcome the potential barrier:

$$
F_{\text {static }}=\frac{2 \pi V_{0}}{a_{x}} .
$$

Now we discuss the behavior of the $1 D$ model at $T=0$, i.e. when $f(t)=0$ in Eq. (4). In this situation the solution of Eq. (4) for $T=0$ is periodic, with period $n a_{x} / v_{s}[14]:$

$$
x\left(t+n a_{x} / v_{s}\right)=x(t)+n a_{x} \quad \text { for integer } n .
$$

Usually $n=1$ for not too small $\eta$.

Elastic instabilities leading to nonadiabatic jumps between metastable states occur for soft cantilever spring constants, in particular when $[24,27]$

$$
k_{x}<-\left.\frac{\partial^{2} V_{t s}}{\partial x^{2}}\right|_{x=x_{m}}, \quad \text { i.e. } \quad \tilde{V}_{0} \equiv \frac{4 \pi^{2} V_{0}}{k_{x} a_{x}^{2}}>1,
$$

where $x_{m}=n a_{x}$ denotes the position of the minima of $V_{t s}$. In this case stick-slip motion, often observed in AFM experiments, is expected and the kinetic friction force is finite in the limit $v_{s} \rightarrow 0$. Conversely, for $\tilde{V}_{0}<1$, uniform sliding occurs and energy dissipation comes only from the viscous term $m \eta v_{s}$, which vanishes for $v_{s} \rightarrow 0$. Notice that the kinetic friction force for $v_{s} \rightarrow 0$ is not equal to the static friction force $F_{\text {static }}$, since it results from dynamical effects and not by the interaction potential $V_{t s}$. The kinetic friction force $F_{\text {fric }}$ is defined as the mean value of the lateral force $F_{x}=k_{x}\left(v_{s} t-x\right)$ over time $[10,14,27]$. By assuming a periodic motion of the type of Eq. (6), $F_{\text {fric }}$ can be written as

$$
F_{\text {fric }}=<F_{x}>\equiv \frac{v_{s}}{n a_{x}} \int_{0}^{n a_{x} / v_{s}} F_{x} d t .
$$

It is easy to show that the definition Eq. (8) is equivalent to calculating the friction force from the energy dissipation $\Delta W$ in one period

$$
\Delta W=m \eta \int_{0}^{n a_{x} / v_{s}} \dot{x}^{2} d t .
$$

The friction force is given by

$$
F_{\text {fric }}=\frac{\Delta W}{n a_{x}} .
$$

Here we extend the model to deal with the motion at zero and finite temperature on a $2 D$ lattice, as done in Refs. [11,27] for $T=0$. The tip-surface interaction is

$$
V_{t s}(x, y)=V_{0} \cos \left(\frac{2 \pi x}{a_{x}}\right) \cos \left(\frac{2 \pi y}{a_{y}}\right),
$$


where $a_{x}$ and $a_{y}$ are the lattice parameters in the $x$ and $y$ directions respectively. When $a_{y}=\sqrt{3} a_{x}$ the substrate has the symmetry of a hexagonal closed-packed lattice. The elastic interaction is

$$
V_{e l}(x, y)=\frac{1}{2} k_{x}\left(x-v_{s} t\right)^{2}+\frac{1}{2} k_{y}\left(y-y_{s}\right)^{2},
$$

where $k_{y}$ denotes the spring constant in the $y$ direction and $y_{s}=$ constant represents the scanning line of the support. The equations of motion can be written in $2 D$ as

$$
\begin{aligned}
& m \ddot{x}+m \eta \dot{x}-V_{0} \sin \left(\frac{2 \pi x}{a_{x}}\right) \cos \left(\frac{2 \pi y}{a_{y}}\right)+k_{x}\left(x-v_{s} t\right)=f_{x}(t) \\
& m \ddot{y}+m \eta \dot{y}-V_{0} \cos \left(\frac{2 \pi x}{a_{x}}\right) \sin \left(\frac{2 \pi y}{a_{y}}\right)+k_{y}\left(y-y_{s}\right)=f_{y}(t) .
\end{aligned}
$$

where $f_{x}$ and $f_{y}$ are independent stochastic forces satisfying the same properties as $f$ in Eq. (4). In this case we also have a component of the lateral force along $y$, i.e. $F_{y}=k_{y}\left(y_{s}-y\right)$. The definition of the friction force in Eq. (8) can be generalized in $2 D$ as

$$
F_{\text {fric }}=\sqrt{\left.\left.<F_{x}\right\rangle^{2}+<F_{y}\right\rangle^{2}}
$$

We have solved the non-linear equations (4) and (13) using a Runge-Kutta 4 algorithm with initial conditions

$$
x(0)=0, \quad \dot{x}(0)=0, \quad y(0)=0, \quad \dot{y}(0)=0 .
$$

and for different values of the scanning velocity $v_{s}$ and of the scanning line $y_{s}$.

\section{ATHERMAL VELOCITY DEPENDENCE OF FRICTION}

At $T=0$ the dynamics can be described by the equations of motion (4) and (13) without the stochastic forces. We choose values of the parameters which are typical of AFM experiments: $m=10^{-10} \mathrm{~kg}, k_{x}=10$ $\mathrm{N} / \mathrm{m}[7,27,29], a_{x}=0.316 \mathrm{~nm}$ (in $2 D$ we set $a_{y}=0.548$ $\mathrm{nm}$, corresponding to the hexagonal-packed structure of $\mathrm{MoS}_{2}(001)$ [27], and $k_{x}=k_{y}$ ), giving a resonance frequency $\sqrt{k_{x} / m}$ of the order of $10^{5} \mathrm{~Hz}$, which is characteristic of AFM experiments. In principle, the corrugation $V_{0}$ of the tip-surface potential depends on the loading force, which is not considered in $1 D$ and $2 D$ models: typically $V_{0}$ ranges from $0.2 \mathrm{eV}$ to $2 \mathrm{eV}$, as found in different studies $[30,31]$. Thus we take $V_{0}=1 \mathrm{eV}$. These values of the parameters give $\tilde{V}_{0}=7$, yielding stick-slip motion $\left(\tilde{V}_{0}>1\right)$ and allowing us to compare directly our results with those of Zwörner et al. in $1 D$ [10]. The time step used in the calculations is $\sim 0.1 \mathrm{~ns}$, a value which is needed to account for the fast oscillations in the underdamped regime. The choice of $\eta$ is quite delicate and it may affect the dynamical behavior of the system. Usually a critical damping, $\eta=2 \sqrt{k_{x} / m}$ [27], is assumed. Here we study the problem for different values of $\eta$, in the underdamped, overdamped and critically damped regime. For each fixed scanning velocity $v_{s}$, we compute the friction force $F_{\text {fric }}$, averaging over many stick-slip periods (usually 10 at $T=0$ and 100 at $T \neq 0$ ), according to Eqs. (8) and (14). The behavior of $F_{\text {fric }}$ as a function of $v_{s}$ in $1 D$ is shown for critical damping in Fig. 1(a) on a linear scale and in Fig. 1(b) on the most commonly used $\log$-log scale [10]. Notice that the log-log scale hides the velocity dependence for small velocities $\left(v_{s}<1.5 \mu \mathrm{m} / \mathrm{s}\right)$, $t)$ where the friction force varies by more than $10 \%$.

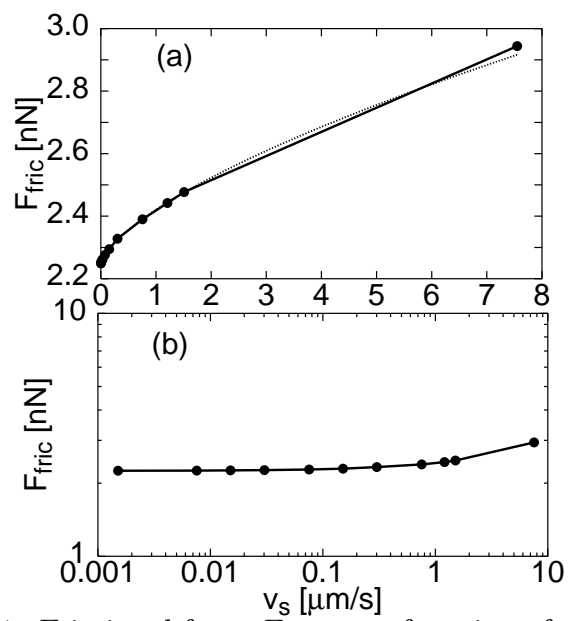

FIG. 1. Frictional force $F_{\text {fric }}$ as a function of sliding velocity $v_{s}$ in the $1 D$ Tomlinson model, plotted on a linear (a) and on a log-log scale (b) for $V_{0}=1 \mathrm{eV}, m=10^{-10} \mathrm{~kg}, k_{x}=10$ $\mathrm{N} / \mathrm{m}, a_{x}=0.316 \mathrm{~nm}\left(\tilde{V}_{0}=7\right)$ and $\eta=2 \sqrt{k_{x} / m} \simeq 6.3 \cdot 10^{5}$ $\mathrm{s}^{-1}$. The increase of $F_{\text {fric }}$ for small velocities is hidden using a log-log scale. The dotted line in (a) is a power-law fit to the data of the form $F_{\text {fric }}-F_{0} \propto v_{s}^{2 / 3}$ for $v_{s}<2 \mu \mathrm{m} / \mathrm{s}$.

The data in Fig. 1(a) can be fitted quite accurately by a power law of the form

$$
F_{\text {fric }}=F_{0}+c v_{s}^{\beta}
$$

with $\beta \simeq 2 / 3$ and $c$ a constant depending on the parameters of the model and on the space dimension.

To our knowledge the athermal velocity dependence of atomistic dry friction has been scarcely investigated up to now: it has been studied in the limit of large velocities [14] and in the context of boundary lubrication [22]. Here we discuss the velocity dependence of dry friction for small scanning velocities, in the stick-slip regime, which is described by Eq. (16). In this case, the value of the exponent $\beta$ can be calculated analytically for the Tomlinson model, yielding $\beta=2 / 3$, as we will show below. The same kind of behavior has been found in the field of elastic manifolds, for the dynamics of charge density waves driven by an electric field [21] and for the motion of a contact line on a heterogeneous surface $[32,33]$. This law characterizes the athermal motion of strongly pinned 
systems $\left(\tilde{V}_{0}>1\right.$ in our terminology $)$, moving at constant velocity.

Considering for simplicity the $1 D$ case and following Ref. [21], we look for a solution $x(t)$ of Eq. (4) in the athermal case $(f(t)=0)$ of the form

$$
x(t)=x_{A}(t)+\theta(t),
$$

where $x_{A}$ is the adiabatic solution of Eq. (4), i.e. the solution for $v_{s} \rightarrow 0$, and $\theta$ is a perturbation. The adiabatic solution satisfies Eq. (4) neglecting the first (inertial) and second (damping) term:

$$
k_{x}\left(x_{A}-v_{s} t\right)=-\frac{2 \pi V_{0}}{a_{x}} \sin \left(\frac{2 \pi x_{A}}{a_{x}}\right)
$$

From Eq. (8) it follows that

$$
\begin{aligned}
F_{\text {fric }}= & <k_{x}\left(v_{s} t-x_{A}-\theta\right)>= \\
& k_{x}<\left(v_{s} t-x_{A}\right)>-k_{x}<\theta>=F_{0}-k_{x}<\theta>,
\end{aligned}
$$

having defined $F_{0} \equiv F_{\text {fric }}\left(v_{s} \rightarrow 0\right)$. Thus, the final goal is to work out the dependence of

$$
<\theta>\equiv \frac{v_{s}}{n a_{x}} \int_{0}^{n a_{x} / v_{s}} \theta(t) d t
$$

on $v_{s}$. First we notice that for $\tilde{V}_{0} \gg 1$ the inertial term $m \ddot{x}$ can be neglected with respect to the damping term $m \eta \dot{x}$ near a slip event. This can be straightforwardly seen in the adiabatic limit. In fact, differentiating Eq. (18) with respect to time we obtain

$$
k_{x} \dot{x}_{A}-k_{x} v_{s}=-\left(\frac{2 \pi}{a_{x}}\right)^{2} V_{0} \cos \left(\frac{2 \pi x_{A}}{a_{x}}\right) \dot{x}_{A},
$$

giving for $\dot{x}_{A}$ and $\ddot{x}_{A}$

$$
z_{A} \equiv \dot{x}_{A}=\frac{k_{x} v_{s}}{k_{x}+\left(\frac{2 \pi}{a_{x}}\right)^{2} V_{0} \cos \left(\frac{2 \pi x_{A}}{a_{x}}\right)}
$$

and

$$
\ddot{x}_{A}=\dot{z}_{A}=\frac{d z_{A}}{d x_{A}} z_{A}=\frac{\left(k_{x} v_{s}\right)^{2}\left(\frac{2 \pi}{a_{x}}\right)^{3} V_{0} \sin \left(\frac{2 \pi x_{A}}{a_{x}}\right)}{\left[k_{x}+\left(\frac{2 \pi}{a_{x}}\right)^{2} V_{0} \cos \left(\frac{2 \pi x_{A}}{a_{x}}\right)\right]^{3}}
$$

respectively. Then the condition

$$
\left|\ddot{x}_{A}\right| \ll \eta\left|\dot{x}_{A}\right|
$$

becomes

$$
\frac{k_{x} v_{s} V_{0}\left(\frac{2 \pi}{a_{x}}\right)^{3}\left|\sin \left(\frac{2 \pi x_{A}}{a_{x}}\right)\right|}{\left[k_{x}+\left(\frac{2 \pi}{a_{x}}\right)^{2} V_{0} \cos \left(\frac{2 \pi x_{A}}{a_{x}}\right)\right]^{2}} \ll \eta .
$$

Since energy dissipation takes place mostly near the fast slip events, we focus on the behavior of Eq. (25) near the slip point $x_{0}$, determined by

$$
\begin{gathered}
\frac{d V_{t o t}}{d x}=k_{x}\left(x-x_{s}\right)+\frac{2 \pi}{a_{x}} V_{0} \sin \left(\frac{2 \pi x}{a_{x}}\right)=0 \\
\frac{d^{2} V_{t o t}}{d x^{2}}=k_{x}+\left(\frac{2 \pi}{a_{x}}\right)^{2} V_{0} \cos \left(\frac{2 \pi x}{a_{x}}\right)=0 .
\end{gathered}
$$

where $V_{t o t}=V_{t s}+V_{e l}$ is the total potential energy. From Eq. (26b) the position $x_{0}$ of the tip right before a slip event is

$$
x_{0}=\frac{a_{x}}{2 \pi} \arccos \left(\tilde{V}_{0}\right) .
$$

Eq. (26a) gives the position $x_{s}^{(0)}$ of the support at the slip point:

$$
x_{s}^{(0)}=\frac{a_{x}}{2 \pi}\left[\sqrt{\tilde{V}_{0}^{2}-1}+\arccos \left(-\frac{1}{\tilde{V}_{0}}\right)\right] .
$$

Near the slip point we can set

$$
x_{A}(t)=x_{0}+\xi_{A}(t)
$$

with

$$
\left|\xi_{A}\right| \ll \frac{a_{x}}{2 \pi} .
$$

Using Eqs. (7) and (26b) and the relations

$$
\begin{aligned}
& \sin \left(\frac{2 \pi x_{A}}{a_{x}}\right) \simeq \sin \left(\frac{2 \pi x_{0}}{a_{x}}\right)+\left(\frac{2 \pi}{a_{x}}\right) \cos \left(\frac{2 \pi x_{0}}{a_{x}}\right) \xi_{A} \\
& \cos \left(\frac{2 \pi x_{A}}{a_{x}}\right) \simeq \cos \left(\frac{2 \pi x_{0}}{a_{x}}\right)-\left(\frac{2 \pi}{a_{x}}\right) \sin \left(\frac{2 \pi x_{0}}{a_{x}}\right) \xi_{A}
\end{aligned}
$$

Eq. (25) becomes

$$
\left|\frac{v_{s}}{\frac{2 \pi}{a_{x}} \sqrt{\tilde{V}_{0}^{2}-1} \xi_{A}^{2}}-\frac{v_{s}}{\left(\tilde{V}_{0}^{2}-1\right) \xi_{A}}\right| \ll \eta .
$$

Since Eq. (30) holds we can neglect the second term with respect to the first, obtaining

$$
\left|\xi_{A}\right| \gg\left(\frac{v_{s} a_{x}}{2 \pi \eta \sqrt{\tilde{V}_{0}^{2}-1}}\right)^{1 / 2} .
$$

Eq. (32) is easily fulfilled for large $\tilde{V}_{0}$ (or large $\eta$ ) and/or small $v_{s}$. For example, with our choice of parameters, yielding $\tilde{V}_{0} \simeq 7$, and $\eta \simeq 6 \cdot 10^{5} \mathrm{~s}^{-1}$, conditions (32) is valid for velocities up to $v_{s} \sim \mu \mathrm{m} / \mathrm{s}$. Having now demonstrated that we can neglect the inertial term, we can expand the equation of motion (without the term $m \ddot{x}$ ) near $x_{0}$ : 


$$
m \eta \dot{\xi}=k_{x} v_{s} \delta t+\frac{1}{2}\left(\frac{2 \pi}{a_{x}}\right)^{3} V_{0} \sin \left(\frac{2 \pi x_{0}}{a_{x}}\right) \xi^{2},
$$

where

$$
\xi=x-x_{0}
$$

and

$$
\delta t=t-t_{0},
$$

$t_{0}$ being the time at which the slip takes place. Following Ref. [21], with the change of variables

$$
\begin{aligned}
\xi & =C^{2} v_{s}^{1 / 3} \chi \\
\delta t & =C v_{s}^{-1 / 3} \tau
\end{aligned}
$$

where $C \equiv \frac{a_{x}}{2 \pi}\left[\frac{V_{0}}{2 m \eta} \sin \left(\frac{2 \pi x_{0}}{a_{x}}\right)\right]^{-1 / 3}$, Eq. (33) takes the form of a Riccati equation:

$$
\frac{d \chi}{d \tau}=\chi^{2}+\frac{k_{x}}{m \eta} \tau
$$

It can be shown [21] that the major contribution to the integral (20) comes from a time $\delta t=\delta t_{s} \equiv t_{1}-t_{0}$ such that $\delta t_{s} \propto v_{s}^{-1 / 3}$. When $t \sim t_{1}$ the solution $\chi(\tau)$ of the Riccati equation has a divergence of the form $\chi(\tau) \sim \frac{1}{\tau_{1}-\tau}$. Note that $\delta t_{s}$ is the slip time, i.e. the time it takes for the tip to go from the metastable position $x=x_{0}$ to the next metastable position $x=x_{1}$. For the adiabatic solution the slip occurs instantaneously, while $\delta t_{s}$ is finite for finite $v_{s}$ and this is responsible for the velocity dependent correction of the friction force. In fact, when $t \sim t_{1}$ $\xi \sim x_{1}-x_{0}$ is of order 1 (e.g. independent of $v_{s}$ ), and $\theta=x-x_{A}=\xi-\xi_{A}$ is of order 1 as well. Thus

$$
<\theta>\simeq \frac{v_{s}}{n a_{x}} \int_{t_{0}}^{t_{1}} \theta(t) d t \propto v_{s}^{2 / 3}
$$

which proves that the exponent $\beta$ appearing in Eq. (16) is $\beta=2 / 3$. This shows that the dependence of friction on velocity is a dynamical effect which is due to the finite (although small) scanning velocity, as it can be seen in Fig. 2, where the tip position $x$ as a function of the support position $x_{s}$ is plotted. The important feature is that the slip events are not instantaneous, as highlighted in the inset of Fig. 2, showing a finite slip time which depends on $v_{s}$. Only if the slip events were really instantaneous a velocity independent friction force would naturally follow from the definition Eq. (8), giving $F_{\text {fric }}=F_{0}$. Therefore, the source of athermal velocity dependence of friction is the non adiabaticity of the motion of the tip for finite $v_{s}$.

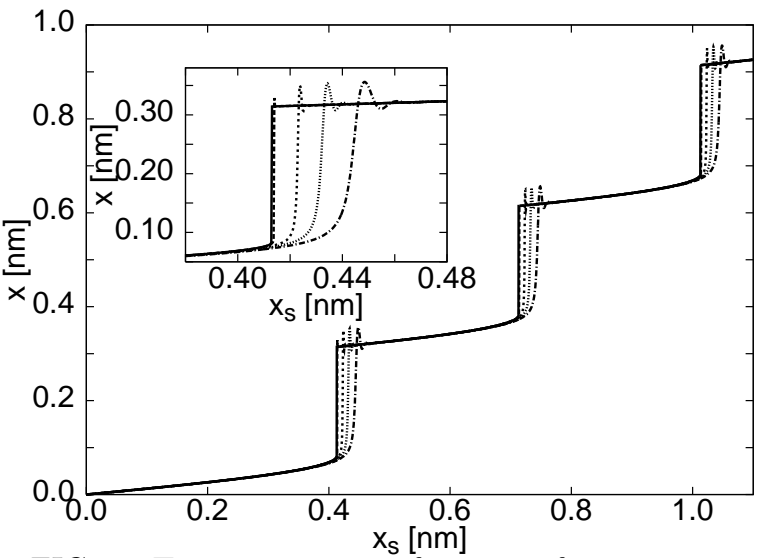

FIG. 2. Tip position as a function of support position in the $1 D$ Tomlinson model for different values of the scanning velocity (from left to right $v_{s}=1.5 \mathrm{~nm} / \mathrm{s}, 15 \mathrm{~nm} / \mathrm{s}, 300 \mathrm{~nm} / \mathrm{s}$, $750 \mathrm{~nm} / \mathrm{s}, 1.5 \mu \mathrm{m} / \mathrm{s}), \eta=2 \sqrt{k_{x} / m}$ and $\tilde{V}_{0}=7$. The inset is a blow up of the region around the first slip event.

Furthermore the slip position tends to move rightwards for increasing $v_{s}$ This means that the integral of $F_{x}=k_{x}\left(x_{s}-x\right)$ over one period

$F_{\text {fric }}=\frac{1}{n a_{x}} \int_{0}^{n a_{x}} F_{x} d x_{s}=\frac{k_{x}}{n a_{x}} \frac{\left(n a_{x}\right)^{2}}{2}-\frac{k_{x}}{n a_{x}} \int_{0}^{n a_{x}} x d x_{s}$

increases with increasing $v_{s}$, since the second term on the right side of Eq. (39) decreases. Fig. 3 shows the slip time $\delta t_{s}$ as a function of $v_{s}$, as measured from the numerical solution of the equation of motion.

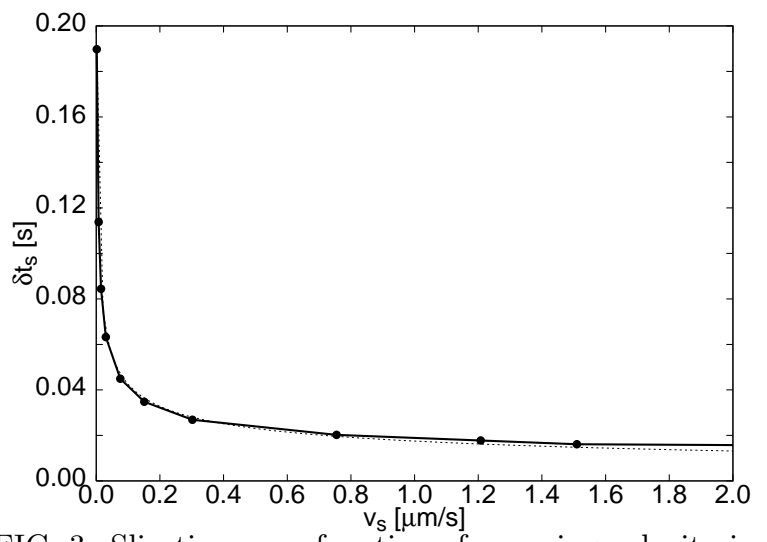

FIG. 3. Slip time as a function of scanning velocity in the $1 D$ Tomlinson model for critical damping and $\tilde{V}_{0}=7$. The points connected by the solid line are obtained by numerical simulations, while the dotted line is a power-law fit to the data of the form $\delta t_{s} \propto v_{s}^{-1 / 3}$.

The behavior of $\delta t_{s}$ is in very good agreement with the scaling relation

$$
\delta t_{s} \propto v_{s}^{-1 / 3}
$$


which is the law expected from the discussion following Eq. (37).

\section{A. Effect of damping}

The effect of the damping parameter on the velocity dependence of friction has not been investigated so far in the literature, because the typical choice is to assume critical damping in order to damp out the fast oscillations of the tip after the slip events and to avoid jumps of the tip of more than one lattice parameter. Nevertheless, it would be desirable to know the dynamical behavior of the tip for a range of values of $\eta$, since experimental situations do not always meet the condition of critical damping. The behavior of $F_{\text {fric }}$ vs. $v_{s}$, for values of $\eta$ ranging from strongly underdamped to strongly overdamped, is reported in Fig. 4.

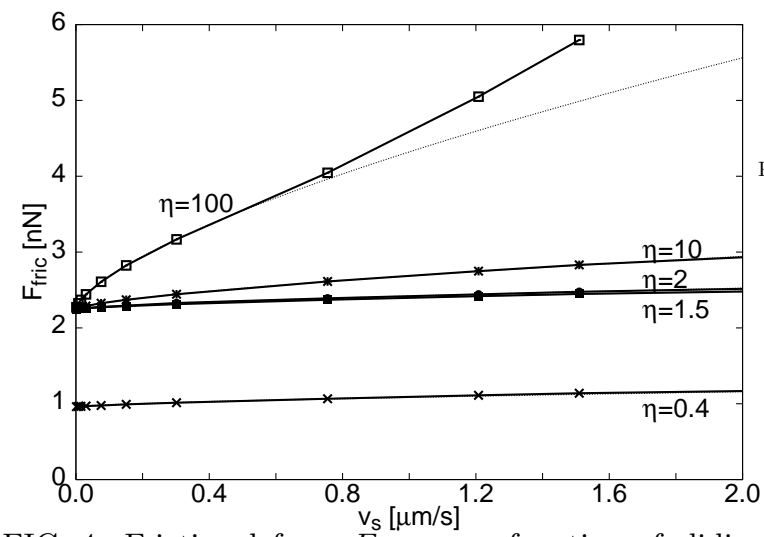

FIG. 4. Frictional force $F_{\text {fric }}$ as a function of sliding velocity $v_{s}$ in the $1 D$ Tomlinson model for $\tilde{V}_{0}=7$ and different values of the damping parameter: from bottom to top $\eta /\left(\sqrt{k_{x} / m}\right)=0.4,1.5,2,10,100$. The dotted lines are fit to the numerical data of the form $F_{\text {fric }}-F_{0} \propto v_{s}^{\beta}$, with $\beta=2 / 3$. In the most underdamped case (lower line) the friction force is lower because the tip performs jumps of two lattice parameters.

All curves start from the same value $F_{0}$, except for very low $\eta$ (see discussion below), and can be fitted by Eq. (16) with the same value of $\beta=2 / 3$, suggesting that the functional form of the velocity dependence of friction is robust with respect to the strength of the damping. The velocity range of validity of Eq. (16) decreases for large $\eta$, because the viscous regime $\left(F_{\text {fric }} \sim m \eta v_{s}\right)$ sets in for smaller values of $v_{s}$ (for example the data in Fig. 4 are fitted up to $v_{s}=1.2 \mu \mathrm{m} / \mathrm{s}$ for $\eta=2 \sqrt{k_{x} / m}$ and up to $v_{s}=0.3 \mu \mathrm{m} / \mathrm{s}$ for $\left.\eta=100 \sqrt{k_{x} / m}\right)$. As expected, at a fixed value of $v_{s}>0, F_{\text {fric }}$ increases with $\eta$, since energy dissipation increases by increasing the damping (see also Eq. (9)). Moreover the value of $c$ in Eq. (16) is larger for larger $\eta$, reflecting the fact that the variation of $F_{\text {fric }}$ is more pronounced for the highest values of $\eta$.

Note that for high damping we find velocity depen- dent friction contrary to the qualitative expectation of Ref. [20]. The authors of Ref. [20] argue that in the overdamped regime the peak velocity of the tip, corresponding to a slip event, is a constant equal to $2 \pi V_{0} /\left(m \eta a_{x}\right)$. This would imply that the amount of energy dissipated, which is proportional to the tip velocity according to Eq. (9), should not depend on $v_{s}$. On the contrary, we find appreciable dependence also in this case. As it can be seen from Fig. 5, the peak velocity of the tip is not a constant, but increases appreciably by increasing $v_{s}$.

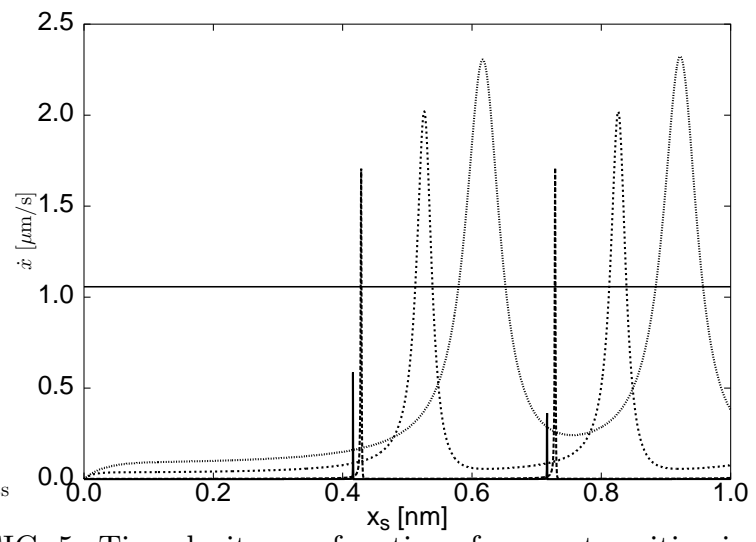

FIG. 5. Tip velocity as a function of support position in the $1 D$ Tomlinson model for different scanning velocities (from left to right $v_{s}=1.5 \mathrm{~nm} / \mathrm{s}, 15 \mathrm{~nm} / \mathrm{s}, 300 \mathrm{~nm} / \mathrm{s}, 750 \mathrm{~nm} / \mathrm{s}$ ) in the overdamped case $\left(\eta=100 \sqrt{k_{x} / m}\right)$ and for $\tilde{V}_{0}=7$. The horizontal line is the value $2 \pi V_{0} /\left(m \eta a_{x}\right)$.

The lower curve in Fig. 4, corresponding to the highly underdamped value $\eta=0.4$, is characterized by a much lower friction force, because the tip in this case can perform jumps with periodicity of two lattice parameters (i.e. $n=2$ in Eq. (6)). This makes the lateral force drop to lower values after a slip event with respect to the critically damped situation, as shown in Fig. 6, resulting in a smaller $F_{0}$. Notice that in Fig. 6 we also plot the so called "mechanistic Tomlinson loop", i.e. $F_{x}=\frac{2 \pi V_{0}}{a_{x}} \sin \left(\frac{2 \pi x}{a_{x}}\right)$ as a function of $x$, as obtained from Eq. (26a). The slip events correspond to transitions between stable branches of this loop.

\section{B. Role of dimensionality}

As already mentioned in the introduction, this problem was recently studied in Ref. [11] using a $2 D$ Tomlinson model, where a velocity dependent friction force was observed even for scanning velocities less than $300 \mathrm{~nm} / \mathrm{s}$. 


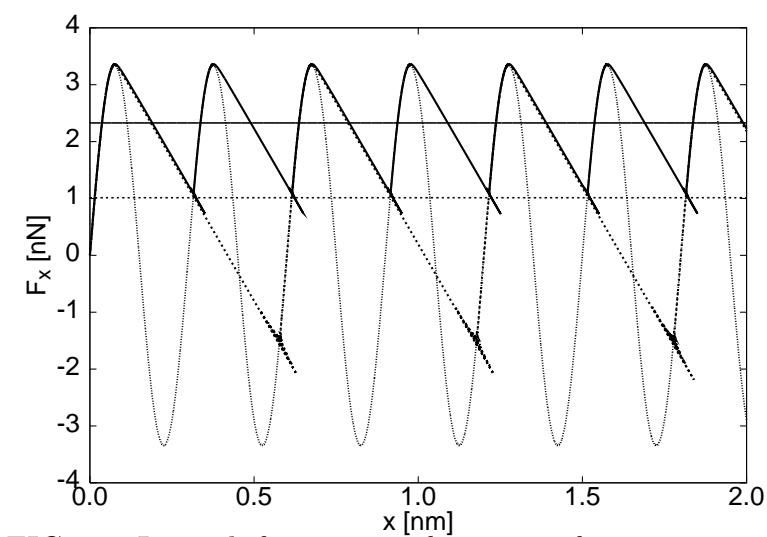

FIG. 6. Lateral force as a function of tip position for two values of the damping parameter: critically damped, $\eta=2 \sqrt{k_{x} / m}$ (solid line) and underdamped, $\eta=0.4 \sqrt{k_{x} / m}$ (dashed line). The reduced corrugation is $\tilde{V}_{0}=7$ and the scanning velocity $v_{s}=300 \mathrm{~nm} / \mathrm{s}$. Notice the presence of jumps with periodicity $2 a_{x}$ in the underdamped case. The upper and lower horizontal lines indicate the friction force for $\eta=2 \sqrt{k_{x} / m}\left(F_{\text {fric }}=2.33 \mathrm{nN}\right)$ and $\eta=0.4 \sqrt{k_{x} / m}$ $\left(F_{\text {fric }}=1.01 \mathrm{nN}\right)$ respectively. The dotted line represents $F_{x}=\frac{2 \pi V_{0}}{a_{x}} \sin \left(\frac{2 \pi x}{a_{x}}\right)$, as obtained from Eq. (26a).

Since for $1 D$ motion no velocity dependence had been previously found in Ref. [10], the authors attributed this dependence to the coupling between the two degrees of freedom of the system. Our results for the $1 D$ Tomlinson model already give a dependence on velocity, and it is interesting to look at the effect of an extra dimension on this dependence. Indeed, as it can be seen in Fig. 7, the behavior of $F_{\text {fric }}$ vs. $v_{s}$ in $2 D$ for different values of the scanning direction $y_{s}$ is very similar to that in $1 D$.

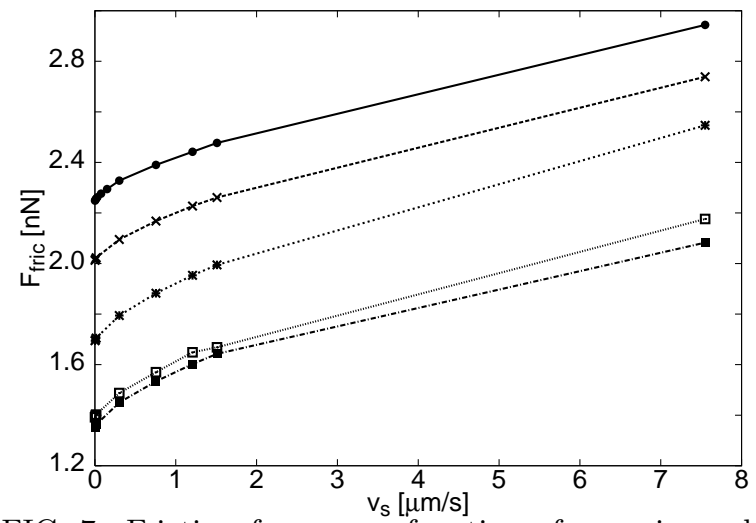

FIG. 7. Friction force as a function of scanning velocity in $1 D$ (upper curve) and $2 D$ Tomlinson model, for critical damping, $\tilde{V}_{0}=7$ and different values of $y_{s}$ (from bottom to top $y_{s}=0.274 \mathrm{~nm}, 0.137 \mathrm{~nm}, 0.1 \mathrm{~nm}$ and $0.05 \mathrm{~nm}$ ).

Thus, in spite of the $2 D$ character of the tip motion, clearly visible in Fig. 8, no dramatic effect of the dimensionality on the velocity dependence of friction can be noticed.

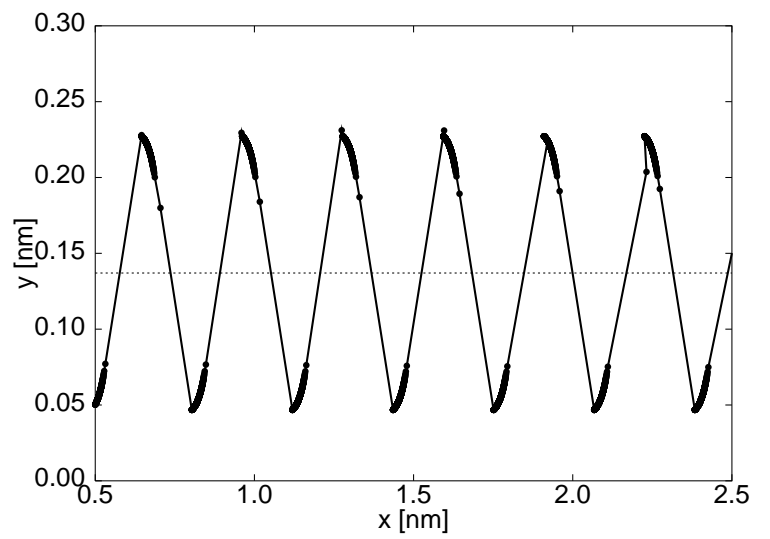

FIG. 8. Trajectory of the tip in the $2 D$ Tomlinson model for critical damping, $\tilde{V}_{0}=7$ and $v_{s}=7.5 \mathrm{~nm} / \mathrm{s}$. The circles connected by the solid line indicate the positions of the tip in the $x y$ plane during the dynamics. The regions where the distribution of points is denser are the sticking domains, where the tip stays predominantly for most of the time. Note that the tip slips from one sticking domain to the other following a zig-zag pattern around the scanning direction (indicated by the dashed line, $y_{s}=0.137 \mathrm{~nm}$ ).

This result is actually not surprising, because the Tomlinson model is a mean-field model and the functional form of constituent relations, such as $F_{\text {fric }}\left(v_{s}\right)$ should not change with dimensionality. Thus Eq. (16) is expected to hold also in $2 D$, with the same exponent $\beta=2 / 3$. The values of the parameters $F_{0}$ and $c$ can be different in $1 D$ and $2 D$. Specifically $F_{0}$ is always lower in $2 D$. In fact, in $1 D$ the tip is necessarily moved along an atom row, while in $2 D$, depending on the scanning line $y_{s}$, the motion of the tip can occur also between atom rows. For the hexagonal lattice we have chosen, the interaction between the tip and the surface is the weakest when $y_{s}=a_{y} / 4$ (bottom curve of Fig. 7 ), while it reaches its maximum value for $y_{s}=0$, which coincides with the $1 D$ case (upper curve of Fig. 7). Since the corrugation of the tip-surface interaction is directly related to the friction force [31], different scanning lines result in different values of friction. This feature allows for example to obtain $2 D$ surface maps in AFM experiments (see for example Ref. [2]). We notice that the absolute variation of $F_{\text {fric }}$ with velocity in the lowest curves of Fig. 7 is more pronounced, thus supporting to a certain extent the claim of Ref. [11]. But it is important that this variation is only due to the different values of the prefactor $c$ in Eq. (16) and not to a change of the exponent $\beta$. Therefore, we can argue that no qualitative differences arise in the velocity dependence of friction in the $2 D$ case and that the common mechanism which produces the observed behavior at $T=0$ can be ascribed to the delayed athermal motion of the tip with respect to the support. 


\section{EFFECT OF THERMAL FLUCTUATIONS}

At finite temperature we integrate numerically the full equations of motion Eqs. (4) and (13). Due the presence of the stochastic forces, the motion of the tip is quite noisy and averages over long trajectories (containing up to 100 periods) have to be considered in order to have a reliable value of the friction force. A typical behavior of the lateral force in $1 D$ for different velocities and critical damping at $T=300 \mathrm{~K}$ is displayed in Fig. 9 .

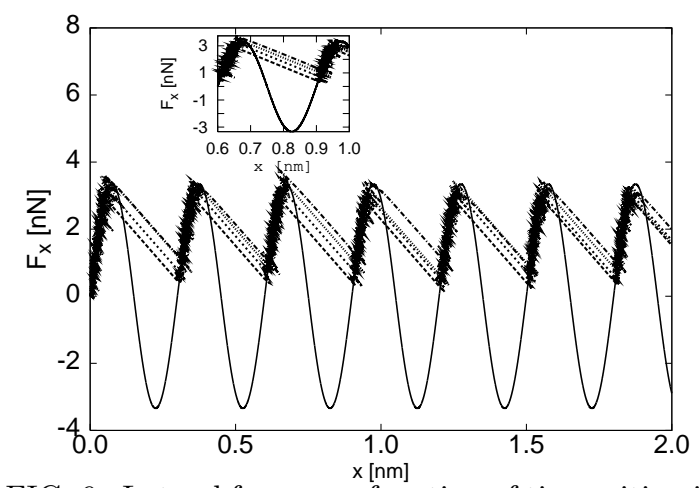

FIG. 9. Lateral force as a function of tip position in the $1 D$ Tomlinson model for critical damping, $T=300 \mathrm{~K}$ and $\tilde{V}_{0}=7$, for different scanning velocities (non-solid lines from bottom to top $\left.v_{s}=1.5 \mathrm{~nm} / \mathrm{s}, 15 \mathrm{~nm} / \mathrm{s}, 300 \mathrm{~nm} / \mathrm{s}, 750 \mathrm{~nm} / \mathrm{s}\right)$. The solid line represents $F_{x}=\frac{2 \pi V_{0}}{a_{x}} \sin \left(\frac{2 \pi x}{a_{x}}\right)$, as obtained from Eq. (26a) (see also Fig. 6). The inset shows a blow up of the region around a slip event.

The height of the maximum for a fixed $v_{s}$ is not constant and the effect of the scanning velocity on the position of the slip is rather pronounced even for small $v_{s}$. In fact, theoretical investigations based on simple analytical approaches in $1 D[9,19]$ and numerical simulations of the $1 D$ Tomlinson model at $T \neq 0$ [19] have shown that temperature is effective in overcoming the energy barriers $\Delta E$, activating jumps of the tip between minima of the total potential energy, for temperatures such that $\Delta E \simeq k_{B} T$. The thermal activation gives rise to a linear logarithmic dependence of friction on velocity for very small scanning velocities [9]:

$$
F_{\text {fric }}-F_{c} \propto \ln \left(v_{s}\right) .
$$

For a larger range of $v_{s}$ the following functional form has been proposed [19]:

$$
F_{\text {fric }}-F_{c} \propto\left|\ln \left(v_{s}\right)\right|^{2 / 3} .
$$

The constant value $F_{c}$ is the lateral force corresponding to a slip event at $T=0$. Eq. (42) is obtained by assuming $\tilde{V}_{0}>1$ and $V_{0} \gg k_{B} T$. As it is shown in Fig. 10, where we compare $F_{\text {fric }}$ vs. $v_{s}$ for $T=0$ and $T=300 \mathrm{~K}$, the main source of velocity dependence of friction is due to thermal fluctuations in the system.

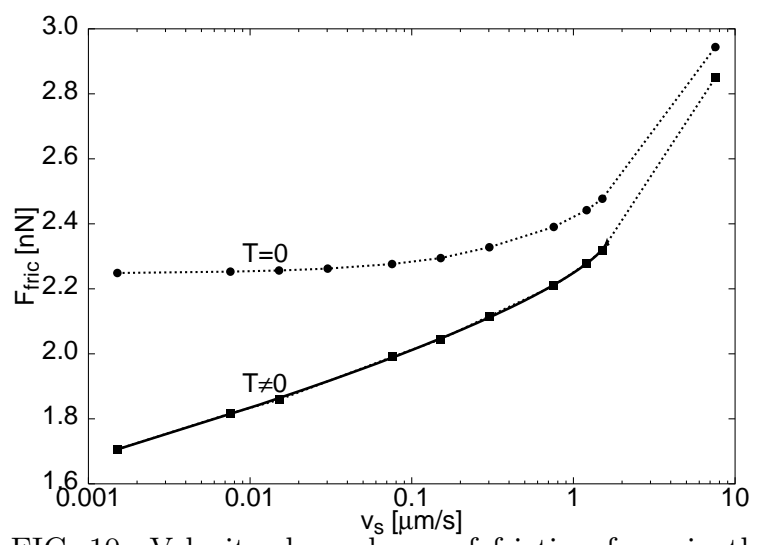

FIG. 10. Velocity dependence of friction force in the $1 D$ Tomlinson model at $T=0$ (upper curve) and $T=300 \mathrm{~K}$ (lower curve) for critical damping and $\tilde{V}_{0}=7$. The solid line is a fit of the data for $T=300 \mathrm{~K}$, using Eq. (42) in the small velocity regime $\left(v_{s}<2 \mu \mathrm{m} / \mathrm{s}\right)$.

The data for $T=300 \mathrm{~K}$ can be fitted by a logarithmic behavior with exponent which is very close to the value $2 / 3$ of Eq. (42). To our knowledge theoretical approaches of velocity dependence of friction at finite temperature have been restricted to $1 D$ models. Here we report results of numerical simulations also for the $2 D$ Tomlinson model, using the same parameters as for the model at $T=0$. Not surprisingly, Fig. 11 shows that the velocity dependence of friction is very similar in $1 D$ and $2 D$, as we have found for $T=0$. We can use Eq. (42) to fit the data of the $2 D$ model as well.

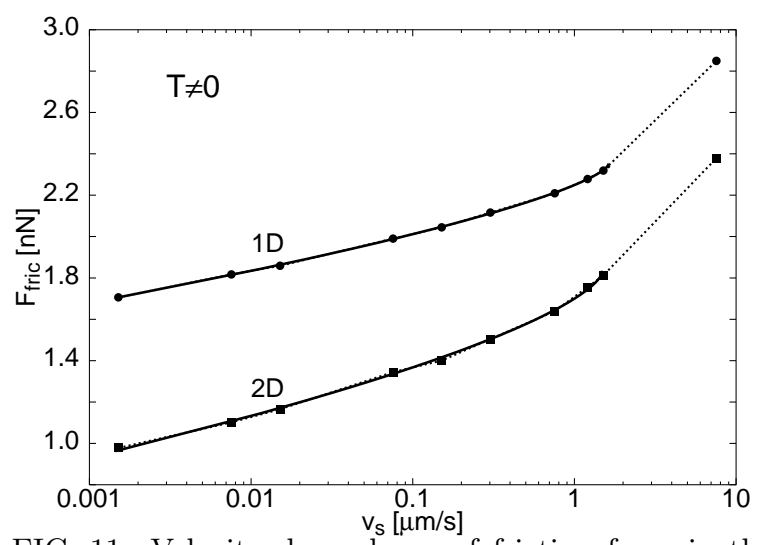

FIG. 11. Velocity dependence of friction force in the $1 D$ (upper curve) and $2 D$ (lower curve) Tomlinson model for $T=300 \mathrm{~K}$, critical damping and $\tilde{V}_{0}=7$. The solid lines are fits to the data using Eq. (42) in the small velocity regime $\left(v_{s}<2 \mu \mathrm{m} / \mathrm{s}\right)$.

In fact, as we have discussed in Sec. III B, the mean field character of the Tomlinson model, preserves the same form of the velocity dependence of energy dissipation.

The different behavior of the friction force with scanning velocity at $T \neq 0$ is due to the activated motion of the tip, which lowers the friction force with respect to 
the athermal situation. This can be easily understood from a sketch of the evolution of the total potential $V_{t o t}$ during the scanning, which is given in Fig. 12.

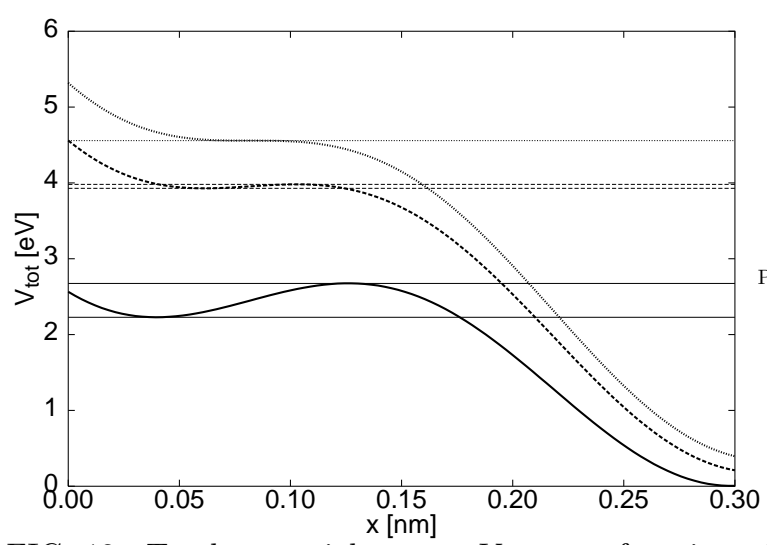

FIG. 12. Total potential energy $V_{t o t}$ as a function of tip position $x$ for three values of the cantilever position $x_{s}$ (from bottom to top $x_{s}=0.287 \mathrm{~nm}, 0.382 \mathrm{~nm}, 0.413 \mathrm{~nm}$ ). The horizontal lines indicate the values of the minimum $\left(V_{\min }\right)$ and the maximum $\left(V_{\max }\right)$ of the potential for each curve. The potential barrier is $\Delta E=V_{\max }-V_{\min }$. The upper curve corresponds to $\Delta E=0$, while the middle curve to the case where $\Delta E \simeq k_{B} T$.

While at $T=0$ a slip event can occur only when the energy barrier $\Delta E$ (i.e. the difference between the maximum and the minimum of $V_{t o t}$ ) vanishes, thermal fluctuations can activate jumps of the tip from a metastable minimum to the next even for finite $\Delta E$, when the cantilever has reached a position which is smaller than the one needed for a slip at $T=0$ : specifically thermal effects start to be significant as soon as $\Delta E$ is few times $k_{B} T$. This has the effect to lower the energy dissipated in a jump, and thus the friction force. The energy barrier is given by

$$
\Delta E(t)=V_{\text {tot }}\left(x_{\max }(t)\right)-V_{\text {tot }}\left(x_{\min }(t)\right)
$$

where $x_{\min }$ and $x_{\max }$ are respectively the positions of a metastable minimum and maximum of $V_{t o t}$.

Fig. 13 compares the velocity dependence of the friction force for three values of $V_{0}$ in the stick-slip regime $\left(V_{0}=0.28 \mathrm{eV}, 0.57 \mathrm{eV}\right.$ and $\left.1 \mathrm{eV}\right)$, with $k_{x}=10 \mathrm{~N} / \mathrm{m}$ (yielding $\tilde{V}_{0}=2,4$ and 7 respectively), both for $T=0$ and $T=300 \mathrm{~K}$. At the smallest scanning velocity considered, in going from $T=0$ to $T=300 \mathrm{~K}, F_{\text {fric }}$ decreases only by a factor 1.2 for $\tilde{V}_{0}=7$, but by a factor 15 for $\tilde{V}_{0}=2$. Indeed, by increasing $\tilde{V}_{0}$, the friction force $F_{\text {fric }}$, in the stick-slip regime, tends to its maximum value $F_{\text {static }}$, and the relative variation in the stick-slip signal decreases. As a consequence, the role of thermally activated processes will be less strong for large $\tilde{V}_{0}$. Moreover, the relative variation of $F_{\text {fric }}$ with $v_{s}$ is much more pronounced for the lowest value of $\tilde{V}_{0}$, and the velocity dependence of friction becomes weaker for larger $\tilde{V}_{0}$.

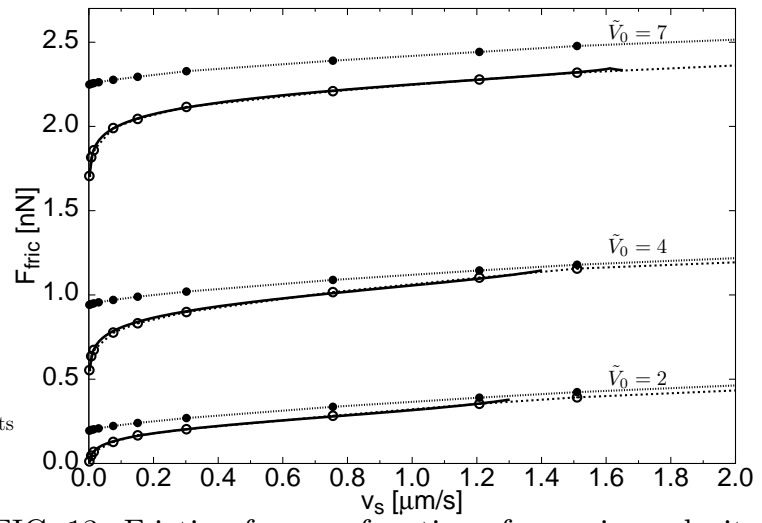

FIG. 13. Friction force as function of scanning velocity for $\tilde{V}_{0}=2, \tilde{V}_{0}=4$ and $\tilde{V}_{0}=7$. The filled circles connected by the dotted lines are the data for $T=0$, while the open circles connected by the dashed lines correspond to the data for $T=300 \mathrm{~K}$. The solid lines are fits to the data at $T=300 \mathrm{~K}$, according to Eq. (44), with exponent $\alpha=0.37$ for $\tilde{V}_{0}=2$, $\alpha=0.56$ for $\tilde{V}_{0}=4$ and $\alpha=0.67 \simeq 2 / 3$ for $\tilde{V}_{0}=7$. The minimum value of the scanning velocity in the plot is $v_{s}=1.5$ $\mathrm{nm} / \mathrm{s}$.

The slope of the curves at $T=300 \mathrm{~K}$ slightly changes by increasing $\tilde{V}_{0}$ and we find that the value $2 / 3$ of the exponent of the logarithmic behavior (Eq. (42)) is recovered for the largest $\tilde{V}_{0}$ we have used. This is in compliance with the approximation used to derive Eq. (42), namely $\tilde{V}_{0}>1$ and $V_{0} \gg k_{B} T$. More generally the data can be fitted by

$$
F_{\text {fric }}-F_{c} \propto\left|\ln \left(v_{s}\right)\right|^{\alpha},
$$

where the exponent $\alpha$ depends on $\tilde{V}_{0}$. In particular, from our data we obtain $\alpha\left(\tilde{V}_{0}=2\right)=0.37, \alpha\left(\tilde{V}_{0}=4\right)=0.57$ and $\alpha\left(\tilde{V}_{0}=7\right)=0.67$. A change of the slope of the velocity-friction curves can also be appreciated in Fig. 1(a) of Ref. [19], where data for different temperatures are presented. This indicates that thermal effects critically depend on the surface corrugation and on temperature.

\section{DISCUSSION AND CONCLUSIONS}

In this paper we have investigated the velocity dependence of sliding friction at the atomic scale within the framework of the Tomlinson model. We have emphasized the role of the athermal processes characterizing the dynamics, which are responsible for a power-law velocity dependence of the friction force at small scanning velocities, while at finite temperature a creep regime takes place, giving rise to a logarithmic behavior of the friction force as a function of velocity. At variance with previous claims in the literature, these dependences apply both in $1 D$ and $2 D$. We have also suggested in a semiquantitative manner in which conditions thermal effects 
are expected to be important for the frictional dynamics. Experimentally, the possibility to observe a velocity dependent frictional force may crucially depend on the nature of the system, which determines the corrugation $V_{0}$, on the stiffness of the cantilever and on the applied loading force, which in turns affects the value of $V_{0}$. Our model is simplified in the sense that the cantilever is treated as a point-like object and the form of energy dissipation, taken into account by introducing a damping term in the equations of motion, is purely phenomenological. Of course, in real situations finite contacts between the tip and the surface are involved and energy dissipation comes into play through more complex mechanisms. However, a simple description based on the Tomlinson model contains the essential ingredients of the problem and can still capture the main dynamical features determining energy dissipation. We expect our study to stimulate further theoretical and experimental work on this issue.

\section{ACKNOWLEDGMENTS}

This work was supported by the Stichting Fundamenteel Onderzoek der Materie (FOM) with financial support from the Nederlandse Organisatie voor Wetenschappelijk Onderzoek (NWO). The authors wish to thank Mikhail Katsnelson, Elisa Riedo, Sergey Krylov and Joost Frenken for interesting and useful discussions.

[1] R. W. Carpick and M. Salmeron, Chem. Rev. 97, 1163 (1997).

[2] E. Gnecco, R. Bennewitz, T. Gyalog and E. Meyer, J. Phys.: Cond. Matt. 13, R619 (2001).

[3] C. M. Mate, G. M. McClelland, R. Erlandsson and S. Chiang, Phys. Rev. Lett. 59, 1942 (1987).

[4] V. N. Koinkar and B. Bhushan, J. Vac. Sci. Technol. A 14, 2378 (1996).

[5] F. Heslot, T. Baumberger, B. Perrin, B. Caroli and C. Caroli, Phys. Rev. E 49, 4973 (1994).

[6] T. Bouhacina, J. P. Aimé, S. Gauthier, D. Michel and V. Heroguez, Phys. Rev. B 56, 7694 (1997).

[7] R. Bennewitz, T. Gyalog, M. Guggisberg, M. Bammerlin, E. Meyer and H.-J. Güntherodt, Phys. Rev. B 60, R11301 (1999).
[8] Y. Hoshi, T. Kawagishi and H. Kawakatsu, Jpn. J. Appl. Phys. 39, 3804 (2000).

[9] E. Gnecco, R. Bennewitz, T. Gyalog, Ch. Loppacher, M. Bammerlin, E. Meyer and H.-J. Güntherodt, Phys. Rev. Lett. 84, 1172 (2000).

[10] O. Zwörner, H. Hölscher, U. D. Schwarz and R. Wiesendanger, Appl. Phys. A 66, S263 (1998).

[11] R. Prioli, A. F. M. Rivas, F. L. Freire Jr., A. O. Caride, Appl. Phys. A 76, 565 (2003).

[12] C. Mak and J. Krim, Phys. Rev. B 58, 5157 (1998).

[13] H. Matsukawa and H. Fukuyama, Phys. Rev. B 49, 17286 (1994).

[14] J. S. Helman, W. Baltensperger and J. A. Hołyst, Phys. Rev. B 49, 3831 (1994).

[15] M. R. Sørensen, K. W. Jacobsen and P. Stoltze, Phys. Rev. B 53, 2101 (1996).

[16] F. Slanina, Phys. Rev. E 59, 3947 (1999).

[17] J. N. Glosli and G. M. McClelland, Phys. Rev. Lett. 70, 1960 (1993).

[18] M. S. Tomassone and J. B. Sokoloff, Phys. Rev. B 60, 4005 (1999).

[19] Y. Sang, M. Dubé and M. Grant, Phys. Rev. Lett. 87, 174301 (2001).

[20] M. O. Robbins and M. H. Müser, in Handbook of Modern Tribology, edited by Bharat Bhushan (CRC press, 2001) [cond-mat/0001056].

[21] D. S. Fisher, Phys. Rev. B 31, 1396 (1985).

[22] M. H. Müser, Phys. Rev. Lett. 89, 224301 (2002).

[23] G. A. Tomlinson, Philos. Mag. 7, 905 (1929).

[24] D. Tománek, W. Zhong and H. Thomas, Europhys. Lett. 15, 887 (1991).

[25] T. Gyalog, M. Bammerlin, R. Lüthi, E. Meyer and H. Tomas, Europhys. Lett. 31, 269 (1995).

[26] H. Hölscher, U. D. Schwarz and R. Wiesendanger, Europhys. Lett. 36, 19 (1996).

[27] H. Hölscher, U. D. Schwarz and R. Wiesendanger, Surf. Sci. 375, 395 (1997).

[28] H. Risken, The Fokker-Planck Equation, 2nd ed., Springer, 1989, chap. 1.

[29] $k_{x}$ corresponds to an effective spring constant given by the series of the stiffness of the contact between the tip and the surface and the spring constant of the cantilever.

[30] E. Riedo, E. Gnecco, R. Bennewitz, E. Meyer and H. Brune, Phys. Rev. Lett. 91, 084502 (2003).

[31] C. Fusco and A. Fasolino, Appl. Phys. Lett. 84, 699 (2004).

[32] J. F. Joanny and M. O. Robbins, J. Chem. Phys. 92, 3206 (1990).

[33] E. Raphaël and P. G. de Gennes, J. Chem. Phys. 90, 7577 (1989). 